\title{
General characteristics of clinical trials for biosimilar drugs
}

\author{
Ahmet AKICI (D), Caner VIZDIKLAR (D)
}

Department of Medical Pharmacology, School of Medicine, Marmara University, Istanbul, Turkey.

\author{
Corresponding Author: Ahmet AKICI
}

E-mail: aakici@marmara.edu.tr

Submitted: 17.01.2021 Accepted: 21.03.2021

\begin{abstract}
Biosimilar is a highly similar product to biological reference medicinal product. The development, licensing and clinical use of these products differ from the implementations of conventional drugs, which were shaped on the concept of equivalence, in various aspects. Negative attitudes towards generic drugs, many of which are thought to be based on lack of knowledge, are still substantial. There is concern that the problem may reach more critical levels in the use of biosimilars, which are known to be more complex compared to generics. Knowing the prominent critical aspects of the development process of the biosimilars may contribute to the solution of this problem that causes significant difficulties in practice.

In all conventional and biological products, the innovative drug research consist of the preclinical phase followed by the successive phases I-IV. The first three phases are carried out before the licensing. The clinical trials required for licensing of generics are limited to bioequivalence studies. However, in biosimilars, this process is more complex due to the nature of biological products. Adequate quality, clinical efficacy and safety data are needed on the basis of comparability of the biosimilar with the reference product. Phase I and III are generally required for biosimilarity, although it may vary per product. Although, these phases are perceived as classical phase studies, they differ considerably from those in the development process of reference drug in terms of design, purpose, content and flexibility. Approaches that do not pay attention to these details sufficiently might cause many problems such as the advantages of biosimilars being limited, ineffective implementation of risk management, loss of trust, delay in product supply, unfair competition, etc. The general characteristics of clinical trials and the details of the subject specifically for biosimilars are mentioned in this review article.
\end{abstract}

Keywords: Biosimilarity, Biological drug, Generic, Clinical trials

\section{INTRODUCTION}

Various professional individuals and organizations, primarily pharmaceutical industry and academic institutions, strive to discover newer and more convenient drugs, introduce them into clinical practice, and form alternative options for treatment, during drug research and development process. All medicinal products for human use, whether being isolated from natural resources or synthesized from chemical or biological materials, are subject to drug research and development processes, including preclinical and clinical trials. Screening and toxicity tests, pharmacodynamic and pharmacokinetic studies in laboratory animals, and other processes such as formulation and stability studies are referred as preclinical research. Candidate medicinal products which successfully completed these processes with sufficient data reach the stage to be tested in humans. Drug development studies conducted on humans within the framework of certain protocols are called clinical trials. A more comprehensive definition, which is also often used by health authorities and other related institutions, is "the studies conducted on humans to reveal or confirm clinical, pharmacological and other pharmacodynamic effects of investigational product(s), define their adverse events/reactions, detect absorption, distribution, metabolism and excretion parameters, and investigate their safety and efficacy" [1]. Although, its history is associated with various cases experienced Before Christ, it can be said that modern drug development processes started from the beginning of the 1900s. Since then, clinical trials have been rapidly shaped through some important medical events, developments, and the regulations introduced

How to cite this article: Akici A, Vizdiklar C. General characteristics of clinical trials for biosimilar drugs. Marmara Med J 2021; 2021; 34(2):89-94. doi: 10.5472/marumj.942752 
as a result of those. Nowadays, it continues to evolve in line with emerging needs and demands. Main characteristics of clinical trials were shaped in the light of past experiences and regulations, and those are valid in universal standards for all drugs. Research on critical products, such as biological drugs (biologics) and their biosimilars, has to include some additional characteristics [2-4]. Although, biologics and their biosimilars are increasingly used in medicine, there seems to be a serious confusion about these products, and their distinguishing features from conventional drugs are not well understood. Regarding choice of generic drugs, a widespread audience among healthcare professionals and patients have negative knowledge, attitudes and behaviours, which are mostly inappropriate [5-7]. Considering this is the case even for the more simply understood conventional drugs, it is concerning that the problem may be even greater for "similars" of biologics, which are known to be more complex $[6,8,9]$. Therefore, lack of knowledge, misinterpretations, and comments about the development process of biosimilars have negative effects not only on the researchers and the parties responsible for the approval/authorization processes of these products, but also on the approach of the various addressees of drug use $[5,6]$. Knowing the critical issues that emerge during development of these products might contribute to solve the problems encountered in practice. In this article, which is mainly focused on the details of clinical research on biosimilar drugs, the main characteristics of clinical trials of drugs are discussed first, in order to facilitate understanding of the subject and to better emphasize its different aspects. Afterwards, the details of the main topic were presented within the framework of the concepts, requirements and regulations specific to biosimilar clinical trials.

\section{Main Characteristics of Clinical Trials}

Drug development around the world is carried out with unprecedentedly high levels of harmonization and strict regulations regarding research and development. At every step of these process, local integration is of particular importance. The intensive intertwined process requires multinational collaborations subject to follow-up and control, and it is tried to be carried out rigorously. Clinical trials can be conducted with the approval of independent scientific ethics committees and with the permission of health authorities. All these stages require sufficient workforce, infrastructure facilities, a long period of time, and large amounts of economic resources. The clinical trial process for a newly innovated drug takes approximately 10 years until approval. Factors such as the new facilities offered by science, technology, and informatics, or the time pressure caused by extraordinary conditions for urgent product needs, such as in the COVID-19 pandemic, provide the basis for the shortening of the aforementioned time period as much as possible. Although the financial burden of research varies depending on the characteristics of the products, generally it is far beyond the easily affordable figures. In other areas of research involving biotechnology and advanced technology, this amount is suggested to reach much higher than that of small-molecule (conventional) drugs, even to the range of one to two billion US dollars [2-4]. Pharmaceutical clinical trials, in which the lucky one among tens of thousands of candidates is determined, are conducted according to strict scientific, technical, administrative and ethical rules, and consists of four successive clinical phases (Figure 1).

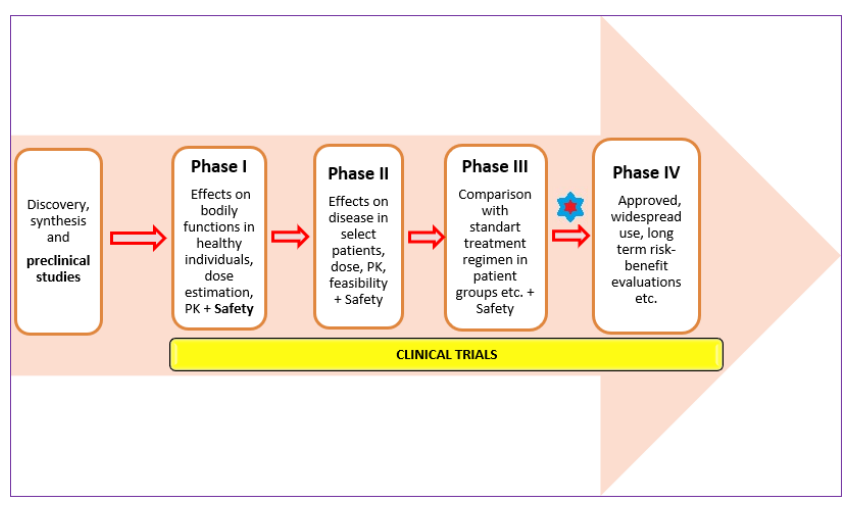

Figure 1. Main characteristics of clinical trial phases

Each of the clinical trial phases, which are carried out for different purposes, proceeds on the results of the previous one within the main framework. Most of the clinical trials are preferably designed as randomized and controlled, depending on the related phase. The first clinical trial phase following preclinical trials is Phase I. Considering the risks potentially involved, it is conducted in specific conditions and mostly in healthy volunteers (except for some cases that would be inappropriate for healthy individuals, which involve patients instead). Phase I aims to determine the preliminary pharmacokinetic properties in humans, pharmacological effects on systems, acute adverse effects, and the resistance of volunteer to the product. Generally, Phase I is carried out in small groups not exceeding 100 people, in specialized units unique to clinical trials, which are equipped for emergency intervention and approved by the related authorities. This stage is followed by Phase II studies, which is conducted on a limited number of patient volunteers (approximately 100500 subjects) and could be regarded as some kind of feasibility studies. Therapeutic dose range, clinical efficacy, safety, pharmacokinetic properties in disease state, and clinical toxicity in that condition are the parameters investigated. After the drug candidate successfully completes Phase II, it goes into Phase III, that is conducted on a large number of patient volunteers (often in the thousands of patients), and mostly in multi-national and multi-center fashion, involving broad participation. In the light of the findings obtained from Phase III trials, the new drug license application for the drug candidate, which successfully completed the aforementioned critical stages, is submitted to the drug authority of the relevant country where the drug is planned to be marketed (e.g., European Medicines Agency [EMA] in Europe, Food and Drug Administration [FDA] in the United States of America [USA], Turkish Medicines and Medical Devices Agency [TMMDA] in Turkey). The common technical 
document (CTD) is used when presenting the information and documents containing preclinical and clinical trial data, along with comprehensive assessment of those. This document needs to be used upon applying to many of the drug authorities, such as that of Turkey, Europe, USA, and Japan. CTD is an internationally agreed format for the standardized submission of applications. This allows reviewing and evaluation to be more systematic [10]. The drug candidate whose application is approved by the authority becomes an "original drug". This new drug is also named as reference drug, as it is a medicinal product that has been licensed with complete dossier. After successfully completing this crucial stage and related other certification and authorization procedures, the product starts to be marketed on a shelf of a pharmacy as a proprietary medicine. Hence, this new drug is allowed to be marketed for the approved indication(s) and posology for the targeted patient audience. However, the licensing of the drug/being on the market does not end the clinical trial process, on the contrary, the research continues with new studies under the name of Phase IV clinical trials. Phase IV trials are vital in many aspects through the dynamic process of drugs. This phase provides access to many crucial information that could not be obtained in previous preclinical and clinical stages. Therefore, these post-marketing clinical trials play decisive roles in the maturation of the drug, its evolution, and its continued presence in the market. Phase IV trials and observational drug research (pharmacoepidemiological studies) which constitute a specific branch of those, in a way question and solidify the reasons for the existence of the drug in the market. Since, the time pressure which was present before licensing is over, the answers to the questions about the drug that could not be prioritized before are planned to be obtained via Phase IV clinical research data in various designs. In this way, important additional information based on evidence is presented to the parties concerned in usage and prescribing of the drug. Except for Phase IV, insurance is mandatory for every clinical trial phase, as well as bioavailability/bioequivalence studies [1-4]. Generics of conventional reference drugs begin to appear when their patent expires. It is assumed that the clinically important risks emerging from potential differences of generics from the reference might mainly originate from the production of the drug, the excipient added to the active substance, and the bioavailability of the product on the basis of the formulation. Owing to this approach, though not suitable for biologics, generics of conventional drugs have the chance to be marketed easily. For a generic drug application, some preclinical baseline data and bioequivalence studies (comparative bioavailability study) is sufficient to be presented to the health authority. If the application is approved, the product starts to be marketed rapidly, as a "generic drug". Standards on bioequivalence were started to be determined in the world around the 1990s, and concrete administrative regulations were quickly implemented. Turkey has necessitated conducting a bioequivalence study for these medicinal products in 1999. Bioequivalence means that "two pharmaceutically equivalent preparations are similar enough to ensure that their bioavailability and hence, their effects are essentially the same in both efficacy and safety after administration at the same molar dose" [1-4]. Both bioavailability/ bioequivalence studies and Phase I clinical trials are conducted in healthcare institutions approved by the regulatory authority, with facilities suitable for emergency intervention and able to meet required standards for each type of study. As in Phase I, bioavailability/bioequivalence studies are carried out in a small number of healthy volunteers. The advantages such as inclusion of limited number of people, shorter duration (as long as the half-life of the drug allows), and well-known and modest designs, shorten the process of generic drugs entering into the market and significantly reduce the costs. This situation may present significant opportunities to reimbursement institutions, pharmaceutical industry, authorities, healthcare professionals, patients, and other parties in terms of competition between a reference drug and its generics $[1,3,4]$.

\section{Main Characteristics of Clinical Trials of Biosimilars}

Biological medicinal product is a product which consists of a biological active substance. In more detail, it can be defined as "medicinal product whose active substance(s) are produced/ purified from a biological source, and whose quality, production process and controls were demonstrated via physicochemical and biological tests" [11]. Clinical research and development process of these drugs are conducted similar to that of conventional drugs. With the patent expiration of a reference biological product, similars of those are developed, which are approved to be marketed only after presenting the obligatory trial data to the health authorities. Biosimilar drug is "a product which is shown to be similar to an approved reference biological product, in terms of physicochemical, in vitro and in vivo biological properties, as well as efficacy, safety and immunogenicity as shown in clinical trials". A more commonly used definition is "a product highly similar to an approved biological medicinal product" $[11,12]$. Biosimilars are not treated the same as conventional generic drugs, due to the large and complex structures of biological products, sensitive multi-step production phases, and their immunogenicity potential. Therefore, it is needed to put forth the similarities with the reference biological product in terms of quality, safety, and efficacy via comparability studies. In this way, satisfactory answers are sought for any uncertainty about residual differences between the reference drug and the biosimilar. Since bioequivalence studies are not sufficient to sort out these uncertainties, Phase I and - in select cases Phase III trials are needed during development of biosimilars, differently from the conventional generics. Even though the primary structures of the reference biological product and the biosimilar are the same, distinct approaches through the production processes of separate manufacturers might lead to any differentiation of the secondary, tertiary and quaternary structures of the products. These potential differences and any related issues are the main reasons for the need for additional clinical trials. The trials need to be sufficiently sensitive in terms of design, method, endpoint and/or volunteer population, in order to detect potential differences between the products. The number and diversity of biotechnological drugs and their biosimilars, and consequently their utilization rates compared to conventional drugs, are consistently rising every year [11-19]. 
The clinical trial phases that biosimilars are subjected, and the clinical data obtained from them are of vital importance to answer questions on development in these drugs, competition between them, whether they provide benefits in terms of cost etc., their possible risks, and their place in clinical use.

The process leading to the registration of the reference biological drug and its biosimilar starts with the pharmaceutical quality studies of both products. In the reference biological drug, this is followed by preclinical studies, Phase I, II and III clinical trials, including analyses of "efficacy and safety, pharmacokinetics and pharmacodynamics, immunogenicity", and a risk management plan. In biosimilar drugs, the steps are Phase I and III clinical trials and risk management plan, respectively. The absence of the need for Phase II and the presence of comprehensive "comparative quality studies" are the main characteristics that distinguish the biosimilar research from the reference [1,4,9-11]. On the other hand, despite sharing the same name, Phase I and III clinical trials of biosimilars differ from the clinical trials of the reference in some aspects, especially in terms of the content. Clinical trials of biosimilar drugs aim to produce data that will contribute to the elimination of residual uncertainties between the reference and its biosimilar. Therefore, expectations from the clinical trial phases of these two products are not the same. Reference biological product trials focus on the production of unique clinical data for a novel product, whereas in biosimilar trials, the partial data production on potential microheterogeneity-related differences from reference drug is of concern $[11,13]$. Without sufficient attention to this critical detail of the content of the research, various demands that diverge from the true aim may be encountered in the process of biosimilar development. The burden of biosimilar clinical trials may increase unnecessarily and introducing these drugs to the market can become difficult.

Comparability data of biosimilars should be obtained using a product that has reached the point of clinical use and has passed the final production process. Pharmacokinetic studies form the basis of clinical comparisons between biosimilar and reference biological medicinal products [11-16]. Phase I clinical trials of biosimilars, unlike that of the reference drug, are not "regular dose estimation studies in which the active substance has been tested in humans for the first time". They generate pharmacokinetic and pharmacodynamic data comparable to the reference. These investigations are conducted by observing the appropriate sample size to meet the requirements of the pharmacokinetic and pharmacodynamic endpoints. These studies, which are generally designed as cross-over or parallel, are preferably carried out in healthy volunteers. In cases where there are risks that are difficult to tolerate in healthy volunteers, studies are carried out in patient volunteers. Another reason for patient volunteer preference is that the biosimilar product is, in some cases, a component of a standard combined treatment regimen. Phase I does not focus directly on efficacy. When the information and evidence on the mechanism of action are sufficiently clear and comprehensible, it can be concluded that a high level of similarity has been reached from a clinical point of view if these two conditions are met: (i) the pharmacokinetic and pharmacodynamic data of the biosimilar in question substantially support the clinical efficacy of the product and (ii) the immunogenicity profiles of the test product and the reference biologic are shown to be similar. In this context, assuming that residual uncertainties in biosimilarity are eliminated, Phase I clinical trials can be deemed sufficient, without requiring further Phase III trials [13-18].

Phase III clinical trials for biosimilars mainly provide efficacy and safety data. These trials are usually conducted as equivalence or non-inferiority studies. These types of studies are not interchangeable, and their results are interpreted quite differently from each other. Choosing the appropriate study type ensures that the hypothesis about the efficacy and safety of the drug is tested correctly and the clinical relevance of the results is accurately evaluated. After the applicant, who will conduct a clinical trial for a biosimilar, submits the planned clinical trial design to the health authority, taking appropriate action according to the consensus to be reached is highly decisive in the rational execution of the process. In the analyses done specifically for the endpoints in an "equivalence trial", H0 hypothesis expresses that biosimilar candidate is "not similar", whereas the $\mathrm{H} 1$ hypothesis means that it is only "similar" (without presenting the argument whether the test product is better or worse than the reference regarding some features in common). While interpreting analyses of a "non-inferiority trial", H0 hypothesis expresses that the tested product "is inferior/does not perform sufficiently", whereas the H1 hypothesis means that it is only "not inferior and is sufficient to prove noninferiority" (but that does not provide any evidence concerning the superiority of test product to the reference drug). If there is valid scientific evidence and prediction that the test product will not be more effective than the reference biological product, non-inferiority studies may be preferred primarily in biosimilar clinical trials, considering the sampling advantage and similar aspects. However, interpretation of the results of these studies is difficult, and should be done carefully, taking any potential confounding into account. Apart from the equivalence and noninferiority trials, there are also "superiority" studies, which are not used in trials of biosimilarity. As the name implies, it can be preferred in the examination of "biosuperior" products with superior efficacy and safety claims than the reference biological drug [11-19].

In phase III clinical trials, it is very important to accurately determine the primary and secondary endpoints. Conducting the trial within a sample large enough and in a suitable population in a randomized controlled manner by choosing the most appropriate study designs mentioned above is also of utmost importance. In this regard, biosimilar clinical trials, which provide comparable efficacy and safety data, are the main elements that guide and help solve problems such as the interchangeability of products, extrapolation, immunogenicity, pharmacovigilance applications and risk management accordingly. By conducting clinical trials within the framework of accurate predictions, the confidence in biosimilars increases and thus, the market entry process of these drugs becomes easier and their place in the market is solidified. For example, safety data obtained from pre-approval clinical trials are one of those 
important details. Especially based on the signs of rare adverse effects, the clinical safety of biosimilar medicinal products, including the ongoing benefit-risk assessment, can be closely monitored following approval. Also, the existing concerns can be addressed or the risks in question can be managed correctly [5-7,11-15].

As with all medicinal products, it is not mandatory to conduct Phase IV clinical trials after registration for biosimilar drugs. Phase IV studies are planned and carried out in the normal course of life, taking the needs of the market into account. In some cases, the parties might plan new study designs earlier, which can be conducted following approval on specific issues that need further clarification (e.g., effects of the product in a large number of patients, or with some alternative treatment options) and make commitments/requests accordingly. Moreover, it might be planned to initiate new pharmacoepidemiological studies on some critical issues, especially those focused on safety. In addition, if pharmacoepidemiological studies are currently being conducted for the reference biological medicinal product, it might be encouraged to include biosimilar(s) in them $[3,11,13]$.

The experience and knowledge gained in a quarter of a century about biological medicinal products, mostly in the last decade, has expanded the range of steps to be taken on biosimilars. In this respect, various flexible approaches and consensus between the parties can emerge more easily in the presence of sufficient scientific evidence on the clinical trial phase preference, design details and similar issues. These approaches, which in essence encourage opening the way for biosimilars, sometimes lead to different steps to be taken by the health authorities in different countries for the same drug. The situation of the medicinal product market, economic or infrastructural factors, other national requirements, different interpretation of the evidence etc. may play a role in that. In order to justify such differences in an indisputable manner, to make them universally consistent and valid, and to minimize confusion and problems that may arise, countries should have functional legislation, healthy communication environments and competent workforce on biosimilars. In addition, the close cooperation of the parties such as the authorities and manufacturers during the clinical research process is of key importance in solving the problems that may arise regarding biosimilars and paving the way for these products. In evaluations related to biosimilars, instead of focusing on a single clinical trial phase, a holistic understanding of the entire process and the related evidence is needed [57,12-16]. Moreover, it is necessary to avoid unfair, nonstandard, non-transparent and shallow approaches, as those concern all stages of biosimilar clinical research and have the risks of adversely affecting both the national and international biosimilar market. In addition, problems of immunogenicity and pharmacovigilance should be made functional in a way that allows for discussion and improvement on scientific grounds, without turning them into a tool of unfair competition. It should be approached cautiously to avoid overstatements and making inferences without the support of appropriate evidence rooting from clinical trial data.
In Turkey, the "Guide on Biosimilar Medicinal Products" could still be regarded as a draft, despite the efforts to update the document in 2017 [11]. Rapidly eliminating this defect is of primary importance for the acceleration of clinical trials of biosimilar drugs and the success of the approval process of these products. In terms of market share, biosimilars follow a parallel trend to reference biologics in recent years, which make up almost one third of the total pharmaceutical market in the world $[12,13,18]$. In order to benefit as much as possible from the advantages offered by using biosimilars in health practice, Turkey is expected to improve their efforts, such as speeding up legal regulation changes, informing parties adequately and extending cooperation options.

\section{Conclusion}

In conclusion, biosimilar drugs will be more commonly encountered in clinical practice. The knowledge and experience gained so far on these drugs, mostly originated from clinical research, is significant. This experience allows further trials regarding new biosimilar drug candidates to be conducted in a flexible way through the context of universal acceptability criteria, rather than being limited by strict rules. It is necessary to adopt a rational approach at all stages of the biosimilar clinical research process, including planning, execution, and data evaluation. Therefore, countries are expected to constitute upto-date and functional legislation, form transparent and strong communication between the parties such as the authorities and the industry, and proceed in the light of versatile and rigorous evaluations by actual experts. The reasons for conducting clinical trials with a product providing sufficient quality assurance should be determined correctly and the data should be obtained accordingly. The reasons for carrying out clinical trials to be conducted with the product providing sufficient quality assurance should be determined correctly, and the data should be obtained accordingly. To demonstrate the quality, efficacy, and safety of these drugs, it is necessary to stick with research and designs that will produce scientific data that will be at universally acceptable levels. In such matters, the parties are expected to convince each other before starting clinical trials. Clinical trials which produce data that do not meet even reasonable minimum expectations should be avoided. Likewise, approaches that would adversely affect the access to biosimilars should be avoided, including over-diversifying research with a defensive medicine approach, demanding convoluted designs, and excessive volunteer participation. In particular, pharmacoeconomic toxicity caused by irrational clinical research demands should be avoided. Approaches that consider these details could prevent unfair competition and loss of trust, provide effective risk management, bring biosimilars with patients in a timely manner, and enable the benefits of these drugs to be properly utilized.

Financial support: The authors have no relevant financial information to disclose.

Conflict of interest: The authors have no potential conflicts to declare. 
Authors' contributions: Conception and design: AA and CV, Formal analysis and investigation: $\mathrm{AA}$ and $\mathrm{CV}$, Writing-review and editing: AA and CV.

\section{REFERENCES}

[1] İlaç ve Biyolojik Ürünlerin Klinik Araştırmaları Hakkında Yönetmelik (13.04.2013 Resmî Gazete Sayısı: 28617) [Regulation on Clinical Trials of Medicines and Biological Products (Turkish Legal Gazette No: 28617)] https://www. titck.gov.tr/mevzuat/ilac-ve-biyolojik-urunlerin-klinikarastirmalari-hakkinda-yonetmelik-271.220.18172740, Accessed: 13-12-2020

[2] Machin D, Day S, Green S. Textbook of Clinical Trials. 2 ed. West Sussex, England: John Wiley and Sons Ltd, 2006, ISBN: 13:978-0-470-01014-3.

[3] Kayaalp SO, (Ed). Klinik Farmakolojinin Esasları ve Temel Düzenlemeler [Principles and Basic Regulations of Clinical Pharmacology]. Updated $5^{\text {th }}$ Edition, Ankara: Pelikan Yayıncllık, 2013. ISBN:978.605.5270-36-0.

[4] Akan H, İlbars H, Çetinkaya NÖ, (Eds), Klinik Araştırmalar Kitabı-2014 [Clinical Trials Book-2014]. Ankara: Bilimsel Tip Yayınevi, ISBN:978.605.4488-48-3.

[5] Barbier L, Simoens S, Vulto AG, Huys I. European stakeholder learnings regarding biosimilars: Part i-improving biosimilar understanding and adoption. BioDrugs 2020;34:783-96. doi: 10.1007/s40259.020.00452-9.

[6] Cohen HP, McCabe D. The importance of countering biosimilar disparagement and misinformation. BioDrugs 2020;34:407-14. doi: 10.1007/s40259.020.00433-y.

[7] Halimi V, Daci A, Netkovska KA, Suturkova L, Babar Z, Grozdanova A. Clinical and regulatory concerns of biosimilars: A review of literature. Int J Environ Res Public Health 2020;17:5800. doi:10.3390/ijerph17165800.

[8] Toklu HZ, Dulger GA, Hidiroglu S, et al. Knowledge and attitudes of the pharmacists, prescribers and patients towards generic drug use in Istanbul - Turkey. Pharmacy Practice 2012;10:199-206.

[9] Oncu S, Bayram D, Aydin V, Isli F, et al. Knowledge, opinions, and attitudes of primary care physicians about generic drugs: a cross-sectional study. Fam Pract 2020; cmaa138. doi:10.1093/ fampra/cmaa138

[10] Turkish Ministry of Health, Turkish Medicines and Medical Devices Agency. "CTD Kılavuzu” [CTD Guide]. https://www. titck.gov.tr/mevzuat/ctd-kilavuzu-271.220.18172815 (Accessed 27-12-2020).

[11] Turkish Ministry of Health, Turkish Medicines and Medical Devices Agency. "Biyobenzer Tibbi Ürünler Hakkında Kılavuz" Taslağ1 [Draft of "Guide on Biosimilar Medicinal Products"], 2017. (https://www.titck.gov.tr/mevzuat/biyobenzer-tibbiurunler-hakkinda-kilavuzu-taslagi-271.220.18173016 Accessed 27-12-2020.

[12] Al-Sabbagh A, Olech E, McClellan JE, Kirchhoff JF. Development of biosimilars. Semin Arthritis Rheum 2016;45(5 Suppl):S11-8.

[13] Çiçin İ. Biyobenzer İlaçlarda Klinik Çalışmalar ve Karşılaştırılabilirlik [Clinical Studies and Comparability in Biosimilar Drugs]. In: Biyoteknolojik İlaçlar [Biotechnological Drugs]. 2 $2^{\text {nd }}$ Edition. Araştırmacı İlaç Firmaları Derneği. İstanbul:Saner Basım Hizm. San. Tic. Ltd. Şti, 2020. ISBN:978625-400-344-8.

[14] European Medicines Agency (EMA). Biosimilar guidelines. https://www.ema.europa.eu/en/human-regulatory/researchdevelopment/scientific-guidelines/multidisciplinary/ multidisciplinary-biosimilar Accessed 27-12-2020.

[15] European Medicines Agency (EMA). Guideline on similar biological medicinal products. (CHMP/437/04 Rev 1). https:// www.ema.europa.eu/documents/scientific-guideline/guidelinesimilar-biological-medicinal-products-rev1_en.pdf. Accessed 27-12-2020.

[16] European Medicines Agency (EMA). Information guide for patients: What I need to know about biosimilar medicines, Information guide for healthcare professionals: Biosimilars in the EU, and animated video on biosimilars in the EU. https://www.ema.europa.eu/en/human-regulatory/overview/ biosimilar-medicines. Accessed 13-12-2020.

[17] Wolff-Holz E, Tiitso K, Vleminckx C, Weise M. Evolution of the EU biosimilar framework: past and future. BioDrugs 2019;33:621-34. doi: 10.1007/s40259.019.00377-y.

[18] Crommelin DJA, Shah VP, Klebovich I, et al. The similarity question for biologicals and non-biological complex drugs. Eur J Pharm Sci 2015;76:10-7. doi: 10.1016/j.ejps.2015.04.010.

[19] Ishii-Watabe A, Takashi Kuwabara T. Biosimilarity assessment of biosimilar therapeutic monoclonal antibodies. Drug Metab Pharmacokinet 2019;34:64-70. doi: 10.1016/j. dmpk.2018.11.004. 